\title{
Explosion of Sun
}

\author{
Alexander Bolonkin, Joseph Friedlander* \\ Strategic Solutions Technology Group, New York, USA \\ Email: abolonkin@juno.com
}

Received November 29, 2012; revised January 5, 2013; accepted January 15, 2013

Copyright (C) 2013 Alexander Bolonkin, Joseph Friedlander. This is an open access article distributed under the Creative Commons Attribution License, which permits unrestricted use, distribution, and reproduction in any medium, provided the original work is properly cited.

\begin{abstract}
The Sun contains $\sim 74 \%$ hydrogen by weight. The isotope hydrogen-1 ( $99.985 \%$ of hydrogen in nature) is a usable fuel for fusion thermonuclear reactions. This reaction runs slowly within the Sun because its temperature is low (relative to the needs of nuclear reactions). If we create higher temperature and density in a limited region of the solar interior, we may be able to produce self-supporting detonation thermonuclear reactions that spread to the full solar volume. This is analogous to the triggering mechanisms in a thermonuclear bomb. Conditions within the bomb can be optimized in a small area to initiate ignition, then spread to a larger area, allowing producing a hydrogen bomb of any power. In the case of the Sun certain targeting practices may greatly increase the chances of an artificial explosion of the Sun. This explosion would annihilate the Earth and the Solar System, as we know them today. The reader naturally asks: Why even contemplate such a horrible scenario? It is necessary because as thermonuclear and space technology spreads to even the least powerful nations in the centuries ahead, a dying dictator having thermonuclear missile weapons can proce (with some considerable mobilization of his military/industrial complex) - an artificial explosion of the Sun and take into his grave the whole of humanity. It might take tens of thousands of people to make and launch the hardware, but only a very few need know the final targeting data of what might be otherwise a weapon purely thought of (within the dictator's defense industry) as being built for peaceful, deterrent use. Those concerned about Man's future must know about this possibility and create some protective system — or ascertain on theoretical grounds that it is entirely impossie. Humanity has fears, justified to greater or lesser degrees, about asteroids, warming of Earthly climate, extinctions, etc. which have very small probability. But all these would leave survivors - nobody thinks that the terrible annihilation of the Solar System would leave a single person alive. That explosion appears possible at the present time. In this paper is derived the "AB-Criterion" which shows conditions wherein the artificial explosion of Sun is possible. The author urges detailed investigation and proving or disproving of this rather horrifying possibility, so that it may be dismissed from mind—or defended against.
\end{abstract}

Keywords: Artificial Explosion of Sun; Annihilation of Solar System; Criterion of Nuclear Detonation; Nuclear Detonation Wave; Detonate Sun; Artificial Supernova

\section{Introduction}

Information about Sun. The Sun is the star at the center of the Solar System. The Earth and other matter (including other planets, asteroids, meteoroids, comets and dust) orbit the Sun, which by itself accounts for about $99.8 \%$ of the solar system's mass. Energy from the Sun-in the form of sunlight - supports almost all life on Earth via photosynthesis, and drives the Earth's climate and weather.

The Sun is composed of hydrogen (about $74 \%$ of its mass, or $92 \%$ of its volume), helium (about $25 \%$ of mass,

\footnotetext{
"J. Friedlander corrected the author's English, wrote together with author Abstract, Sections 8, 10 ("Penetration into Sun" and "Results"), and wrote Section 11 "Discussion" as the solo author.
}

$7 \%$ of volume), and trace quantities of other elements. The Sun has a spectral class of G2V. G2 implies that it has a surface temperature of approximately $5500 \mathrm{~K}$ (or approximately 9600 degrees Fahrenheit/5315 Celsius), Sunlight is the main source of energy to the surface of Earth. The solar constant is the amount of power that the Sun deposits per unit area that is directly exposed to sunlight. The solar constant is equal to approximately 1370 watts per square meter of area at a distance of one AU from the Sun (that is, on or near Earth). Sunlight on the surface of Earth is attenuated by the Earth's atmosphere so that less power arrives at the surface-closer to 1000 watts per directly exposed square meter in clear conditions when the Sun is near the zenith. 
The Sun is about halfway through its main-sequence evolution, during which nuclear fusion reactions in its core fuse hydrogen into helium. Each second, more than 4 million tonnes of matter are converted into energy within the Sun's core, producing neutrinos and solar radiation; at this rate, the sun will have so far converted around 100 earth-masses of matter into energy. The Sun will spend a total of approximately 10 billion years as a main sequence star.

The core of the Sun is considered to extend from the center to about 0.2 solar radii. It has a density of up to $150,000 \mathrm{~kg} / \mathrm{m}^{3}$ (150 times the density of water on Earth) and a temperature of close to 13,600,000 kelvins (by contrast, the surface of the Sun is close to 5785 kelvins $\left(1 / 2350^{\text {th }}\right.$ of the core $\left.)\right)$. Through most of the Sun's life, energy is produced by nuclear fusion through a series of steps called the $p$ - $p$ (proton-proton) chain; this process converts hydrogen into helium. The core is the only location in the Sun that produces an appreciable amount of heat via fusion: the rest of the star is heated by energy that is transferred outward from the core. All of the energy produced by fusion in the core must travel through many successive layers to the solar photosphere before it escapes into space as sunlight or kinetic energy of particles [1].

About $3.4 \times 10^{38}$ protons (hydrogen nuclei) are converted into helium nuclei every second (out of about $\sim 8.9$ $\times 10^{56}$ total amount of free protons in Sun), releasing energy at the matter-energy conversion rate of 4.26 million tonnes per second, 383 yottawatts $\left(383 \times 10^{24} \mathrm{~W}\right)$ or $9.15 \times 10^{10}$ megatons of TNT per second. This corresponds to extremely low rate of energy production in the Sun's core-about $0.3 \mu \mathrm{W} / \mathrm{cm}^{3}$, or about $6 \mu \mathrm{W} / \mathrm{kg}$. For comparison, an ordinary candle produces heat at the rate $1 \mathrm{~W} / \mathrm{cm}^{3}$, and human body - at the rate of $1.2 \mathrm{~W} / \mathrm{kg}$. Use of plasma with similar parameters as solar interior plasma for energy production on Earth is completely impractical - as even a modest $1 \mathrm{GW}$ fusion power plant would require about 170 billion tonnes of plasma occupying almost one cubic mile. Thus all terrestrial fusion reactors require much higher plasma temperatures than those in Sun's interior to be viable.

The rate of nuclear fusion depends strongly on density (and particularly on temperature), so the fusion rate in the core is in a self-correcting equilibrium: a slightly higher rate of fusion would cause the core to heat up more and expand slightly against the weight of the outer layers, reducing the fusion rate and correcting the perturbation; and a slightly lower rate would cause the core to cool and shrink slightly, increasing the fusion rate and again reverting it to its present level.

The high-energy photons (gamma and X-rays) released in fusion reactions are absorbed in only few millimeters of solar plasma and then re-emitted again in random direction (and at slightly lower energy) — so it takes a long time for radiation to reach the Sun's surface. Estimates of the "photon travel time" range from as much as 50 million years to as little as 17,000 years. After a final trip through the convective outer layer to the transparent "surface" of the photosphere, the photons escape as visible light. Each gamma ray in the Sun's core is converted into several million visible light photons before escaping into space. Neutrinos are also released by the fusion reactions in the core, but unlike photons they very rarely interact with matter, so almost all are able to escape the Sun immediately.

This reaction is very slowly because the solar temperatute is very lower of Coulomb barrier.

The Sun's current age, determined using computer models of stellar evolution and nucleocosmochronology, is thought to be about 4.57 billion years.

Astronomers estimate that there are at least 70 sextillion $\left(7 \times 10^{22}\right)$ stars in the observable universe. That is 230 billion times as many as the 300 billion in the Milky Way [2].

Atmosphere of Sun. The parts of the Sun above the photosphere are referred to collectively as the solar atmosphere. They can be viewed with telescopes operating across the electromagnetic spectrum, from radio through visible light to gamma rays, and comprise five principal zones: the temperature minimum, the chromosphere, the transition region, the corona, and the heliosphere.

The chromosphere, transition region, and corona are much hotter than the surface of the Sun; the reason why is not yet known. But their density is low.

The coolest layer of the Sun is a temperature minimum region about $500 \mathrm{~km}$ above the photosphere, with a temperature of about $4000 \mathrm{~K}$.

Above the temperature minimum layer is a thin layer about 2,000 km thick, dominated by a spectrum of emission and absorption lines. It is called the chromosphere from the Greek root chroma, meaning color, because the chromosphere is visible as a colored flash at the beginning and end of total eclipses of the Sun. The temperature in the chromosphere increases gradually with altitude, ranging up to around 100,000 K near the top.

Above the chromosphere is a transition region in which the temperature rises rapidly from around 100,000 $\mathrm{K}$ to coronal temperatures closer to one million $\mathrm{K}$. The increase is because of a phase transition as helium within the region becomes fully ionized by the high temperatures. The transition region does not occur at a well-defined altitude. Rather, it forms a kind of nimbus around chromospheric features such as spicules and filaments, and is in constant, chaotic motion. The transition region is not easily visible from Earth's surface, but is readily observable from space by instruments sensitive to the far ultraviolet portion of the spectrum. 
The corona is the extended outer atmosphere of the Sun, which is much larger in volume than the Sun itself. The corona merges smoothly with the solar wind that fills the solar system and heliosphere. The low corona, which is very near the surface of the Sun, has a particle density of $10^{14} \mathrm{~m}^{-3}-10^{16} \mathrm{~m}^{-3}$. (Earth's atmosphere near sea level has a particle density of about $2 \times 10^{25} \mathrm{~m}^{-3}$.) The temperature of the corona is several million kelvin. While no complete theory yet exists to account for the temperature of the corona, at least some of its heat is known to be from magnetic reconnection [3].

Physical characteristics of Sun: Mean diameter is $1.392 \times 10^{6} \mathrm{~km}$ (109 Earths). Volume is $1.41 \times 10^{18} \mathrm{~km}^{3}$ $\left(1,300,000\right.$ Earths). Mass is $1.988435 \times 10^{30} \mathrm{~kg}$ $\left(332,946\right.$ Earths). Average density is $1408 \mathrm{~kg} / \mathrm{m}^{3}$. Surface temperature is $5785 \mathrm{~K}(0.5 \mathrm{eV})$. Temperature of corona is $5 \mathrm{MK}(0.43 \mathrm{keV})$. Core temperature is $\sim 13.6$ MK $(1.18 \mathrm{keV})$. Sun radius is $R=696 \times 10^{3} \mathrm{~km}$, solar gravity $g_{c}=274 \mathrm{~m} / \mathrm{s}^{2}$. Photospheric composition of Sun (by mass): Hydrogen 73.46\%; Helium 24.85\%; Oxygen $0.77 \%$; Carbon 0.29\%; Iron 0.16\%; Sulphur 0.12\%; Neon $0.12 \%$; Nitrogen $0.09 \%$; Silicon $0.07 \%$; Magnesium $0.05 \%$.

Sun photosphere has thickness about $7 \times 10^{-4} R(490$ $\mathrm{km}$ ) of Sun radius $R$, average temperature $5.4 \times 10^{3} \mathrm{~K}$, and average density $2 \times 10^{-7} \mathrm{~g} / \mathrm{cm}^{3}\left(n=1.2 \times 10^{23} \mathrm{~m}^{-3}\right)$. Sun convection zone has thickness about $0.15 R$, average temperature $0.25 \times 10^{6} \mathrm{~K}$, and average density $5 \times 10^{-7}$ $\mathrm{g} / \mathrm{cm}^{3}$. Sun intermediate (radiation) zone has thickness about $0.6 R$, average temperature $4 \times 10^{6} \mathrm{~K}$, and average density $10 \mathrm{~g} / \mathrm{cm}^{3}$. Sun core has thickness about $0.25 R$, average temperature $11 \times 10^{6} \mathrm{~K}$, and average density 89 $\mathrm{g} / \mathrm{cm}^{3}$.

Detonation is a process of combustion in which a supersonic shock wave is propagated through a fluid due to an energy release in a reaction zone. This self-sustained detonation wave is different from a deflagration, which propagates at a subsonic rate (i.e., slower than the sound speed in the material itself).

Detonations can be produced by explosives, reactive gaseous mixtures, certain dusts and aerosols.

The simplest theory to predict the behavior of detonations in gases is known as Chapman-Jouguet $(\mathrm{CJ})$ theory, developed around the turn of the 20th century. This theory, described by a relatively simple set of algebraic equations, models the detonation as a propagating shock wave accompanied by exothermic heat release. Such a theory confines the chemistry and diffusive transport processes to an infinitely thin zone.

A more complex theory was advanced during World War II independently by Zel'dovich, von Neumann, and Doering. This theory, now known as ZND theory, admits finite-rate chemical reactions and thus describes a detonation as an infinitely thin shock wave followed by a zone of exothermic chemical reaction. In the reference frame in which the shock is stationary, the flow following the shock is subsonic. Because of this, energy release behind the shock is able to be transported acoustically to the shock for its support. For a self-propagating detonation, the shock relaxes to a speed given by the Chapman-Jouguet condition, which induces the material at the end of the reaction zone to have a locally sonic speed in the reference frame in which the shock is stationary. In effect, all of the chemical energy is harnessed to propagate the shock wave forward.

Both CJ and ZND theories are one-dimensional and steady. However, in the 1960 s experiments revealed that gas-phase detonations were most often characterized by unsteady, three-dimensional structures, which can only in an averaged sense be predicted by one-dimensional steady theories. Modern computations are presently making progress in predicting these complex flow fields. Many features can be qualitatively predicted, but the multi-scale nature of the problem makes detailed quantitative predictions very difficult [1-4].

\section{Statement of Problem, Main Idea and Our Aim}

The present solar temperature is far lower than needed for propagating a runaway thermonuclear reaction. In Sun core the temperature is only $\sim 13.6 \mathrm{MK}(0.0012$ $\mathrm{MeV}$ ). The Coulomb barrier for protons (hydrogen) is more then $0.4 \mathrm{MeV}$. Only very small proportions of core protons take part in the thermonuclear reaction (they use a tunnelling effect). Their energy is in balance with energy emitted by Sun for the Sun surface temperature $5785 \mathrm{~K}(0.5 \mathrm{eV})$.

We want to clarify: If we create a zone of limited size with a high temperature capable of overcoming the Coulomb barrier (for example by insertion of a thermonuclear warhead) into the solar photosphere (or lower), can this zone ignite the Sun's photosphere (ignite the Sun's full load of thermonuclear fuel)? Can this zone selfsupport progressive runaway reaction propagation for a significant proportion of the available thermonuclear fuel?

If it is possible, researchers can investigate the problems: What will be the new solar temperature? Will this be metastable, decay or runaway? How long will the transformed Sun live, if only a minor change? What the conditions will be on the Earth?

Why is this needed?

As thermonuclear and space technology spreads to even the least powerful nations in the decades and centuries ahead, a dying dictator having thermonuclear weapons and space launchers can produce (with some considerable mobilization of his military/industrial complex)- 
the artificial explosion of the Sun and take into his grave the whole of humanity.

It might take tens of thousands of people to make and launch the hardware, but only a very few need know the final targeting data of what might be otherwise a weapon purely thought of (within the dictator's defense industry) as being built for peaceful, "business as usual" deterrent use. Given the hideous history of dictators in the twentieth century and their ability to kill technicians who had outlived their use (as well as major sections of entire populations also no longer deemed useful) we may assume that such ruthlessness is possible.

Given the spread of suicide warfare and self-immolation as a desired value in many states, (in several cultures-think Berlin or Tokyo 1945, New York 2001, Tamil regions of Sri Lanka 2006) what might obtain a century hence? All that is needed is a supportive, obedient defense complex, a "romantic" conception of mass death as an ideal - even a religious ideal — and the realization that his own days at power are at a likely end. It might even be launched as a trump card in some (to us) crazy internal power struggle, and plunged into the Sun and detonated in a mood of spite by the losing side. "Burn baby burn"!

A small increase of the average Earth's temperature over $0.4 \mathrm{~K}$ in the course of a century created a panic in humanity over the future temperature of the Earth, resulting in the Kyoto Protocol. Some stars with active thermonuclear reactions have temperatures of up to $30,000 \mathrm{~K}$. If not an explosion but an enchanced burn results the Sun might radically increase in luminosity for say - a few hundred years. This would suffice for an average Earth temperature of hundreds of degrees over $0^{\circ} \mathrm{C}$. The oceans would evaporate and Earth would bake in a Venus like greenhouse, or even lose its' atmosphere entirely.

Thus we must study this problem to find methods of defense from human induced Armageddon.

The interested reader may find needed information in [4-9].

\section{Theory Estimations and Computation}

1) Coulomb barrier (repulsion). Energy is needed for thermonuclear reaction may be computed by equations

$$
\begin{aligned}
& E=\frac{k Z_{1} Z_{2} e^{2}}{r}=2.3 \times 10^{-28} \frac{Z_{1} Z_{2}}{r}[\mathrm{~J}] \\
& \text { or } E=\frac{k Z_{1} Z_{2} e}{r}=1.44 \times 10^{-9} \frac{Z_{1} Z_{2}}{r}[\mathrm{eV}], \\
& \text { where } \quad r=r_{1}+r_{2}, \quad r_{i}=(1.2 \div 1.5) \times 10^{-15} \sqrt[3]{A_{i}}
\end{aligned}
$$

where $E$ is energy needed for forcing contact between two nuclei, $\mathrm{J}$ or $\mathrm{eV} ; k=9 \times 10^{9}$ is electrostatic constant, $\mathrm{N} \cdot \mathrm{m}^{2} / \mathrm{C}^{2} ; Z$ is charge state; $e=1.6 \times 10^{-19}$ is charge of proton, $\mathrm{C} ; r$ is distance between nucleus centers, $\mathrm{m} ; r_{i}$ is radius of nucleus, $\mathrm{m} ; A=Z+N$ is nuclei number, $N$ is number neutrons into given $(i=1,2)$ nucleus.

The computations of average temperature (energy) for some nucleus are presented in Table $\mathbf{1}$ below. We assume that the first nucleus is moving; the second (target) nucleus is motionless.

In reality the temperature of plasma may be significantly lower than in table 1 because the nuclei have different velocity. Parts of them have higher velocity (see Maxwell distribution of nuclei speed in plasma), some of the nuclei do not (their energy are summarized), and there are tunnel effects. If the temperature is significantly lower, then only a small part of the nuclei took part in reaction and the fuel burns very slowly. This case we have-happily in the present day Sun where the temperature in core has only $0.0012 \mathrm{MeV}$ and the Sun can burn at this rate for billions of years $[5,6]$.

The ratio between temperatures in $\mathrm{eV}$ and in $\mathrm{K}$ is

$$
T_{K}=1.16 \times 10^{4} T_{e}, \quad T_{e}=0.86 \times 10^{-4} T_{K} .
$$

2) The energy of a nuclear reaction. The energy and momentum conservation laws define the energetic relationships for a nuclear reaction $[1,2]$.

When a reaction $A(a, b) B$ occurs, the quantity

$$
Q=\left[\left(M_{A}+M_{a}\right)-\left(M_{B}+M_{b}\right)\right] c^{2},
$$

where $M_{i}$ are the masses of the particles participating in the reaction and $c$ is the speed of light, Q is the reaction energy.

Usually mass defects $\Delta M$ are used, instead of masses, for computing $Q$ :

$$
Q=\left(\Delta M_{A}+\Delta M_{a}\right)-\left(\Delta M_{B}+\Delta M_{b}\right) .
$$

The mass defect is the quantity $\Delta M=M-A$ where $M$ is the actual mass of the particle (atom), $A$ is the socalled mass number, i.e. the total number of nucleons (protons and neutrons) in the atomic nucleus. If $M$ is expressed in atomic mass units (a.m.u.) and $A$ is assigned the same unit, then $\Delta M$ is also expressed in a.m.u. One a.m.u. represent $1 / 12$ of the ${ }^{12} \mathrm{C}$ nuclide mass and equals $1.6605655 \times 10^{-27} \mathrm{~kg}$. For calculations of reaction energies it is more convenient to express $\Delta M$ in kilo-electronvolts: a.m.u. $=931501.59 \mathrm{keV}$.

Employing the mass defects, one can handle numbers that are many times smaller than the nuclear masses or the binding energies.

Table 1. Columb barrier of some nuclei pairs.

\begin{tabular}{cccccccc}
\hline Reaction & $\begin{array}{c}E, \\
\mathrm{MeV}\end{array}$ & Reaction & $\begin{array}{c}E, \\
\mathrm{MeV}\end{array}$ & Reaction & $\begin{array}{c}E, \\
\mathrm{MeV}\end{array}$ & Reaction & $\begin{array}{c}E, \\
\mathrm{MeV}\end{array}$ \\
\hline $\mathrm{p}+\mathrm{p}$ & 0.53 & $\mathrm{~T}+\mathrm{p}$ & 0.44 & ${ }^{6} \mathrm{~L}+\mathrm{p}$ & 1.13 & ${ }^{13} \mathrm{C}+\mathrm{p}$ & 1.9 \\
$\mathrm{D}+\mathrm{p}$ & 0.47 & $\mathrm{D}+\mathrm{d}$ & 0.42 & ${ }^{7} \mathrm{Be}+\mathrm{p}$ & 1.5 & $\begin{array}{c}{ }^{12} \mathrm{C}+ \\
{ }^{4} \mathrm{He}\end{array}$ & 3.24 \\
\hline
\end{tabular}


Kinetic energy may be released during the course of a reaction (exothermic reaction) or kinetic energy may have to be supplied for the reaction to take place (endothermic reaction). This can be calculated by reference to a table of very accurate particle rest masses (see http://physics.nist.gov/PhysRefData/Compositions/index. $\mathrm{html}$ ). The reaction energy (the "Q-value") is positive for exothermal reactions and negative for endothermal reactions.

The other method calculate of thermonuclear energy is in [1]. For a nucleus of atomic number $Z$, mass number $A$, and Atomic mass $M(Z, A)$, the binding energy is

$$
Q=\left[Z M\left({ }^{1} \mathrm{H}\right)+(A-Z) m_{n}-M(Z, A)\right] c^{2},
$$

where $M\left({ }^{1} H\right)$ is mass of a hydrogen atom and $m_{n}$ is mass of neutron. This equation neglects a small correction due to the binding energy of the atomic electrons.

The binding energy per nucleus $Q / A$, varies only slightly in the range of $7-9 \mathrm{MeV}$ for nuclei with $A>12$.

The binding energy can be approximately calculated from Weizsacker's semiempirical formula:

$$
\begin{aligned}
& Q=a_{v} A-a_{s} A^{2 / 3}-a_{c} Z(Z-1) A^{-1 / 3} \\
& -a_{s y m}(A-2 Z)^{2} / A+\delta
\end{aligned}
$$

where $\delta$ accounts for pairing of like nucleons and has the value $+a_{p} A^{-3 / 4}$ for $Z$ and $N$ both even, $-a_{p} A^{-3 / 4}$ for $Z$ and $N$ both odd, and zero otherwise ( $A$ odd). The constants in this formula must be adjusted for the best agreement with data: typical values are $a_{v}=15.5 \mathrm{MeV}, a_{s}=16.8 \mathrm{MeV}, a_{c}$ $=0.72 \mathrm{MeV}, a_{s y m}=23 \mathrm{MeV}$, and $a_{p}=34 \mathrm{MeV}$.

The binding energy per nucleon of the helium-4 nucleus is unusually high, because the He-4 nucleus is doubly magic. (The He-4 nucleus is unusually stable and tightly-bound for the same reason that the helium atom is inert: each pair of protons and neutrons in He- 4 occupies a filled 1s nuclear orbital in the same way that the pair of electrons in the helium atom occupies a filled $1 \mathrm{~s}$ electron orbital). Consequently, alpha particles appear frequently on the right hand side of nuclear reactions $[7,8]$.

The energy released in a nuclear reaction can appear mainly in one of three ways:

- kinetic energy of the product particles.

- emission of very high energy photons, called gamma rays.

- some energy may remain in the nucleus, as a metastable energy level.

When the product nucleus is metastable, this is indicated by placing an asterisk (“*”) next to its atomic number. This energy is eventually released through nuclear decay.

If the reaction equation is balanced, that does not mean that the reaction really occurs. The rate at which reactions occur depends on the particle energy, the particle flux and the reaction cross section.

In the initial collision which begins the reaction, the particles must approach closely enough so that the short range strong force can affect them. As most common nuclear particles are positively charged, this means they must overcome considerable electrostatic repulsion before the reaction can begin. Even if the target nucleus is part of a neutral atom, the other particle must penetrate well beyond the electron cloud and closely approach the nucleus, which is positively charged. Thus, such particles must be first accelerated to high energy, for example by very high temperatures, on the order of millions of degrees, producing thermonuclear reactions

Also, since the force of repulsion is proportional to the product of the two charges, reactions between heavy nuclei are rarer, and require higher initiating energy, than those between a heavy and light nucleus; while reactions between two light nuclei are commoner still.

Neutrons, on the other hand, have no electric charge to cause repulsion, and are able to affect a nuclear reaction at very low energies. In fact at extremely low particle energies (corresponding, say, to thermal equilibrium at room temperature), the neutron's de Broglie wavelength is greatly increased, possibly greatly increasing its capture cross section, at energies close to resonances of the nuclei involved. Thus low energy neutrons may be even more reactive than high energy neutrons [9].

3) Distribution of thermonuclear energy between particles. In most cases, the result of thermonuclear reaction is more than one product. As you see in Table 2 that may be "He" and neutron or proton. The thermonuclear energy distributes between them in the following manner:

$$
\begin{aligned}
& \text { From } E=E_{1}+E_{2}=\frac{m_{1} V_{1}^{2}}{2}+\frac{m_{2} V_{2}^{2}}{2}, \quad m_{1} V_{1}=m_{2} V_{2}, \\
& \text { we have } \frac{E_{1}}{E}=\frac{m_{2}}{m_{1}+m_{2}}=\frac{\mu_{2}}{\mu_{1}+\mu_{2}}, \quad E_{2}=E-E_{1},
\end{aligned}
$$

where $m$ is particle mass, $\mathrm{kg} ; V$ is particle speed, $\mathrm{m} / \mathrm{s} ; E$ is particle energy, $\mathrm{J} ; \mu=m_{i} / m_{p}$ is relative particle mass. Lower indexes " 1,2 " are number of particles.

After some collisions the energy $E=k T$ (temperature) of different particles may be closed to equal.

4) The power density produced in thermonuclear reaction may be computed by the equation

$$
P=E n_{1} n_{2}\langle\sigma v\rangle,
$$

where $E$ is energy of single reaction, $\mathrm{eV}$ or $\mathrm{J} ; n_{1}$ is density (number particles in $\mathrm{cm}^{3}$ ) the first component; $n_{2}$ is density (number particles in $\mathrm{cm}^{3}$ ) the second component; $\langle\sigma v\rangle$ is reaction rate, in $\mathrm{cm}^{3} / \mathrm{s} ; \sigma$ is cross section of reaction, $\mathrm{cm}^{2}, 1$ barn $=10^{-24} \mathrm{~cm}^{2} ; v$ is speed of the first component, $\mathrm{cm} / \mathrm{s} ; P$ is power density, $\mathrm{eV} / \mathrm{cm}^{3}$ or $\mathrm{J} / \mathrm{cm}^{3}$. Cross section of reaction before $\sigma_{\max }$ very strongly 
Table 2. Exothermic thermonuclear reactions.

\begin{tabular}{|c|c|c|c|c|c|c|c|c|c|}
\hline № & Reaction & $\begin{array}{c}\text { Energy of } \\
\text { reaction } \mathrm{MeV}\end{array}$ & $\begin{array}{c}\sigma_{\max } \text { barn } \\
E \leq 1 \mathrm{MeV}\end{array}$ & $\begin{array}{c}E \text { of } \sigma_{\max } \\
\mathrm{MeV}\end{array}$ & № & $\begin{array}{c}\text { Reaction } \\
\mathrm{MeV}\end{array}$ & $\begin{array}{c}\text { Energy of } \\
\text { Reaction } \mathrm{MeV}\end{array}$ & $\begin{array}{c}\sigma_{\max } \text { barn } \\
E \leq 1 \mathrm{MeV}\end{array}$ & $\begin{array}{c}E \text { of } \sigma_{\max } \\
\mathrm{MeV}\end{array}$ \\
\hline 1 & $\mathrm{p}+\mathrm{p} \rightarrow \mathrm{d}+\mathrm{e}^{+}+\mathrm{v}$ & 2.2 & $10^{-23}$ & - & 15 & $\mathrm{~d}+{ }^{6} \mathrm{Li} \rightarrow{ }^{7} \mathrm{Li}+\mathrm{p}$ & 5.0 & 0.01 & 1 \\
\hline 2 & $\mathrm{p}+\mathrm{d} \rightarrow{ }^{3} \mathrm{He}+\gamma$ & 5.5 & $10^{-6}$ & - & 16 & $\mathrm{~d}+{ }^{6} \mathrm{Li} \rightarrow 2{ }^{4} \mathrm{He}$ & 22.4 & 0.026 & 0.60 \\
\hline 3 & $\mathrm{p}+\mathrm{t} \rightarrow{ }^{4} \mathrm{He}+\gamma$ & 19.7 & $10^{-6}$ & - & 17 & $\mathrm{~d}+{ }^{7} \mathrm{Li} \rightarrow 2{ }^{4} \mathrm{He}+\mathrm{n}$ & 15.0 & $10^{-3}$ & 0.2 \\
\hline 4 & $d+d \rightarrow t+p$ & 4.0 & 0.16 & 2 & 18 & $\mathrm{p}+{ }^{9} \mathrm{Be} \rightarrow 2{ }^{4} \mathrm{He}+\mathrm{d}$ & 0.56 & 0.46 & 0.33 \\
\hline 5 & $\mathrm{~d}+\mathrm{d} \rightarrow{ }^{3} \mathrm{He}+\mathrm{n}$ & 3.3 & 0.09 & 1 & 19 & $\mathrm{p}+{ }^{9} \mathrm{Be} \rightarrow{ }^{6} \mathrm{Li}+{ }^{4} \mathrm{He}$ & 2.1 & 0.34 & 0.33 \\
\hline 6 & $\mathrm{~d}+\mathrm{d} \rightarrow{ }^{4} \mathrm{He}+\gamma$ & 24 & - & - & 20 & $\mathrm{p}+{ }^{11} \mathrm{~B} \rightarrow 3{ }^{4} \mathrm{He}$ & 8.7 & 0.6 & 0.675 \\
\hline 7 & $\mathrm{~d}+\mathrm{t} \rightarrow{ }^{4} \mathrm{He}+\mathrm{n}$ & 17.6 & 5 & 0.13 & 21 & $\mathrm{p}+{ }^{15} \mathrm{~N} \rightarrow{ }^{12} \mathrm{C}+{ }^{4} \mathrm{He}$ & 5.0 & 0.6 & 1.2 \\
\hline 8 & $\mathrm{t}+\mathrm{d} \rightarrow{ }^{4} \mathrm{He}+\mathrm{n}$ & 17.6 & 5 & 0.195 & 22 & $\mathrm{~d}+{ }^{6} \mathrm{Li} \rightarrow{ }^{7} \mathrm{Be}+\mathrm{n}$ & 3.4 & 0.01 & 0.3 \\
\hline 9 & $\mathrm{t}+\mathrm{t} \rightarrow{ }^{4} \mathrm{He}+2 \mathrm{n}$ & 11.3 & 0.1 & 1 & 23 & ${ }^{3} \mathrm{He}+\mathrm{t} \rightarrow{ }^{4} \mathrm{He}+\mathrm{d}$ & 14.31 & 0.7 & $\approx 1$ \\
\hline 10 & $\mathrm{~d}+{ }^{3} \mathrm{He} \rightarrow{ }^{4} \mathrm{He}+\mathrm{p}$ & 18.4 & 0.71 & 0.47 & 24 & ${ }^{3} \mathrm{H}+{ }^{4} \mathrm{He} \rightarrow{ }^{7} \mathrm{Li}+\gamma$ & 2.457 & $7 \cdot 10^{-5}$ & $\approx 3$ \\
\hline 11 & ${ }^{3} \mathrm{He}+{ }^{3} \mathrm{He} \rightarrow{ }^{4} \mathrm{He}+2 \mathrm{p}$ & 12.8 & - & - & 25 & ${ }^{3} \mathrm{H}+\mathrm{d} \rightarrow{ }^{4} \mathrm{He}$ & 17.59 & $5 \cdot 10^{-4}$ & $\approx 2$ \\
\hline 12 & $\mathrm{n}+{ }^{6} \mathrm{Li} \rightarrow{ }^{4} \mathrm{He}+\mathrm{t}$ & 4,8 & 2.6 & 0.26 & 26 & ${ }^{12} \mathrm{C}+\mathrm{p} \rightarrow{ }^{13} \mathrm{~N}+\gamma$ & 1.944 & $10^{-6}$ & 0.46 \\
\hline 13 & $\mathrm{p}+{ }^{6} \mathrm{Li} \rightarrow{ }^{4} \mathrm{He}+{ }^{3} \mathrm{He}$ & 4,0 & $10^{-4}$ & 0.3 & 27 & ${ }^{13} \mathrm{C}+\mathrm{p} \rightarrow{ }^{14} \mathrm{~N}+\gamma$ & 7.55 & $10^{-4}$ & 0.555 \\
\hline 14 & $\mathrm{p}+{ }^{7} \mathrm{Li} \rightarrow 2{ }^{4} \mathrm{He}+\gamma$ & 17.3 & $6 \cdot 10^{-3}$ & 0.44 & 28 & ${ }^{3} \mathrm{He}+{ }^{4} \mathrm{He} \rightarrow{ }^{7} \mathrm{Be}+\gamma$ & 1.587 & $10^{-6}$ & $\approx 8$ \\
\hline
\end{tabular}

Here are: $\mathrm{p}\left(\right.$ or $\left.{ }^{1} \mathrm{H}\right)$ - proton, d (or $\mathrm{D}$, or $\left.{ }^{2} \mathrm{H}\right)$ - deuterium, t (or $\mathrm{T}$, or $\left.{ }^{3} \mathrm{H}\right)$ - tritium, $\mathrm{n}$ — neutron, He-helium, Li-lithium, Be-beryllium, B-barium, $\mathrm{C}$ - carbon, $\mathrm{N}$-hydrogen, $v$-neutrino, $\gamma$-gamma radiation.

depends from temperature and it is obtainable by experiment. They can have the maximum resonance. For very high temperatures the $\sigma$ may be close to the nuclear diameter.

The terminal velocity of the reaction components (electron and ions) are

$$
\begin{gathered}
v_{T e}=\left(k T_{e} / m_{e}\right)^{1 / 2}=4.19 \times 10^{7} T_{e}^{1 / 2} \cdot \mathrm{cm} / \mathrm{s}, \\
v_{T i}=\left(k T_{i} / m_{i}\right)^{1 / 2}=9.79 \times 10^{7}\left(T_{i} / \mu_{i}\right)^{1 / 2} \cdot \mathrm{cm} / \mathrm{s},
\end{gathered}
$$

where $T$ is temperature in $\mathrm{eV} ; \mu_{i}=m_{i} / m_{p}$ is ratio of ion mass to proton mass.

The sound velocity of ions is

$$
v=\left(\frac{\gamma z k T_{k}}{m_{i}}\right)^{1 / 2},
$$

where $\gamma \approx(1.2-1.4)$ is adiabatic coefficient; $z$ is number of charge ( $z=1$ for $p$ ), $T_{k}$ is plasma temperature in $\mathrm{K} ; m_{i}$ is mass of ion.

The deep of penetration of outer radiation into plasma is

$$
d=5.31 \times 10^{5} n_{e}^{-1 / 3},[\mathrm{~cm}]
$$

where $n_{e}$ is number of electrons in unit of volume.

In internal plasma detonation there is no loss in radiation because the plasma reflects the radiation.

\section{Possible Thermonuclear Reactions to Power a Hypothetical Solar Explosion}

The Sun mass is $\sim 74 \%$ hydrogen and $25 \%$ helium.

Possibilities exist for the following self-supporting nu- clear reactions in the hydrogen medium: proton chain reaction, $\mathrm{CNO}$ cycle, Triple-alpha process, Carbon burning process, Neon burning process, Oxygen burning process, Silicon burning process.

For our case of particular interest (a most probable candidate) the proton-proton chain reaction. It is more exactly the reaction $p+p$.

The proton-proton chain reaction is one of several fusion reactions by which stars convert hydrogen to helium, the primary alternative being the CNO cycle. The proton-proton chain dominates in stars the size of the Sun or less.

The first step involves the fusion of two hydrogen nuclei ${ }^{1} \mathrm{H}$ (protons) into deuterium ${ }^{2} \mathrm{H}$, releasing a positron and a neutrino as one proton changes into a neutron.

$$
{ }^{1} \mathrm{H}+{ }^{1} \mathrm{H} \rightarrow{ }^{2} \mathrm{H}+\mathrm{e}^{+}+v_{\mathrm{e}} .
$$

with the neutrinos released in this step carrying energies up to $0.42 \mathrm{MeV}$.

The positron immediately annihilates with an electron, and their mass energy is carried off by two gamma ray photons.

$$
\mathrm{e}^{+}+\mathrm{e}^{-} \rightarrow 2 \gamma+1.02 \mathrm{MeV} .
$$

After this, the deuterium produced in the first stage can fuse with another hydrogen to produce a light isotope of helium, ${ }^{3} \mathrm{He}$ :

$$
{ }^{2} \mathrm{H}+{ }^{1} \mathrm{H} \rightarrow{ }^{3} \mathrm{He}+\gamma+5.49 \mathrm{MeV} .
$$

From here there are three possible paths to generate helium isotope ${ }^{4} \mathrm{He}$. In pp1 helium-4 comes from fusing two of the helium-3 nuclei produced; the pp2 and pp3 
branches fuse ${ }^{3} \mathrm{He}$ with a pre-existing ${ }^{4} \mathrm{He}$ to make Beryllium-7. In the Sun, branch pp1 takes place with a frequency of $86 \%$, pp 2 with $14 \%$ and pp3 with $0.11 \%$. There is also an extremely rare pp4 branch.

\section{1) The pp I branch}

$$
{ }^{3} \mathrm{He}+{ }^{3} \mathrm{He} \rightarrow{ }^{4} \mathrm{He}+{ }^{1} \mathrm{H}+{ }^{1} \mathrm{H}+12.86 \mathrm{MeV}
$$

The complete pp I chain reaction releases a net energy of $26.7 \mathrm{MeV}$. The pp I branch is dominant at temperatures of 10 to 14 megakelvins (MK). Below $10 \mathrm{MK}$, the $\mathrm{PP}$ chain does not produce much ${ }^{4} \mathrm{He}$.

2) The pp II branch

\begin{tabular}{ccc}
\hline${ }^{3} \mathrm{He}+{ }^{4} \mathrm{He}$ & $\rightarrow$ & ${ }^{7} \mathrm{Be}+\gamma$ \\
${ }^{7} \mathrm{Be}+\mathrm{e}^{-}$ & $\rightarrow$ & ${ }^{7} \mathrm{Li}+v_{\mathrm{e}}$ \\
${ }^{7} \mathrm{Li}+{ }^{1} \mathrm{H}$ & $\rightarrow$ & ${ }^{4} \mathrm{He}+{ }^{4} \mathrm{He}$ \\
\hline
\end{tabular}

The pp II branch is dominant at temperatures of 14 to $23 \mathrm{MK} .90 \%$ of the neutrinos produced in the reaction ${ }^{7} \mathrm{Be}\left(\mathrm{e}^{-}, v_{\mathrm{e}}\right)^{7} \mathrm{Li}^{*}$ carry an energy of $0.861 \mathrm{MeV}$, while the remaining $10 \%$ carry $0.383 \mathrm{MeV}$ (depending on whether lithium-7 is in the ground state or an excited state, respectively).

\section{3) The pp III branch}

\begin{tabular}{ccc}
\hline${ }^{3} \mathrm{He}+{ }^{4} \mathrm{He}$ & $\rightarrow$ & ${ }^{7} \mathrm{Be}+\gamma$ \\
${ }^{7} \mathrm{Be}+{ }^{1} \mathrm{H}$ & $\rightarrow$ & ${ }^{8} \mathrm{~B}+\gamma$ \\
${ }^{8} \mathrm{~B}$ & $\rightarrow$ & ${ }^{8} \mathrm{Be}+\mathrm{e}^{+}+v_{\mathrm{e}}$ \\
${ }^{8} \mathrm{Be}$ & $\leftrightarrow$ & ${ }^{4} \mathrm{He}+{ }^{4} \mathrm{He}$ \\
\hline
\end{tabular}

The pp III chain is dominant if the temperature exceeds $23 \mathrm{MK}$.

The pp III chain is not a major source of energy in the Sun (only $0.11 \%$ ), but was very important in the solar neutrino problem because it generates very high energy neutrinos (up to $14.06 \mathrm{MeV}$ ).

\section{4) The pp IV or hep}

This reaction is predicted but has never been observed due to its great rarity (about 0.3 parts per million in the Sun). In this reaction, Helium-3 reacts directly with a proton to give helium-4, with an even higher possible neutrino energy (up to $18.8 \mathrm{MeV}$ ).

$$
{ }^{3} \mathrm{He}+{ }^{1} \mathrm{H} \rightarrow{ }^{4} \mathrm{He}+v_{\mathrm{e}}+\mathrm{e}^{+}
$$

\section{5) Energy release}

Comparing the mass of the final helium-4 atom with the masses of the four protons reveals that 0.007 or $0.7 \%$ of the mass of the original protons has been lost. This mass has been converted into energy, in the form of gamma rays and neutrinos released during each of the individual reactions.
The total energy we get in one whole chain is

$$
4{ }^{1} \mathrm{H} \rightarrow{ }^{4} \mathrm{He}+26.73 \mathrm{MeV} \text {. }
$$

Only energy released as gamma rays will interact with electrons and protons and heat the interior of the Sun. This heating supports the Sun and prevents it from collapsing under its own weight. Neutrinos do not interact significantly with matter and do not help support the Sun against gravitational collapse. The neutrinos in the ppI, ppII and ppIII chains carry away the $2.0 \%, 4.0 \%$ and $28.3 \%$ of the energy respectively.

This creates a situation in which stellar nucleosynthesis produces large amounts of carbon and oxygen but only a small fraction of these elements is converted into neon and heavier elements. Both oxygen and carbon make up the ash of helium burning. Those nuclear resonances sensitively are arranged to create large amounts of carbon and oxygen, has been controversially cited as evidence of the anthropic principle.

About $34 \%$ of this energy is carried away by neutrinos. That reaction is part of solar reaction, but if initial temperature is high, the reaction becomes an explosion.

The detonation wave works a short time. That supports the reactions (12)-(13). They produce energy up to 1.44 $\mathrm{MeV}$. The reactions (12)-(14) produce energy up to 5.8 $\mathrm{MeV}$. But after detonation wave and the full range of reactions the temperature of plasma is more than the temperature needed to pass the Coulomb barrier and the energy of explosion increases by 20 times [10-12].

\section{Theory of Detonation}

The one dimensional detonation wave may be computed by equations (see Figure 1):

1) Law of mass

$$
\frac{D}{V_{1}}=\frac{v}{V_{3}},
$$

where $D$-speed of detonation, $\mathrm{m} / \mathrm{s} ; v$-speed of ion sound, $\mathrm{m} / \mathrm{s}$ about the front of detonation wave (Equation (11)); $V_{1}, V_{3}$ specific density of plasma in points 1,3 respectively, $\mathrm{kg} / \mathrm{m}^{3}$.

2) Law of momentum

$$
p_{1}+\frac{D^{2}}{V_{1}}=p_{3}+\frac{v^{2}}{V_{3}},
$$

where $p_{1}, p_{3}$ are pressures, $\mathrm{N} / \mathrm{m}^{2}$, in point 1,3 respectively.

3) Law of energy

$$
E_{3}-E_{1}=Q+0.5\left(p_{3}+p_{1}\right)\left(V_{1}-V_{3}\right),
$$

where $E_{3}, E_{1}$-internal energy, $\mathrm{J} / \mathrm{kg}$, of mass unit in point 3,1 respectively, $Q$ is nuclear energy, $\mathrm{J} / \mathrm{kg}$.

4) Speed of detonation is 


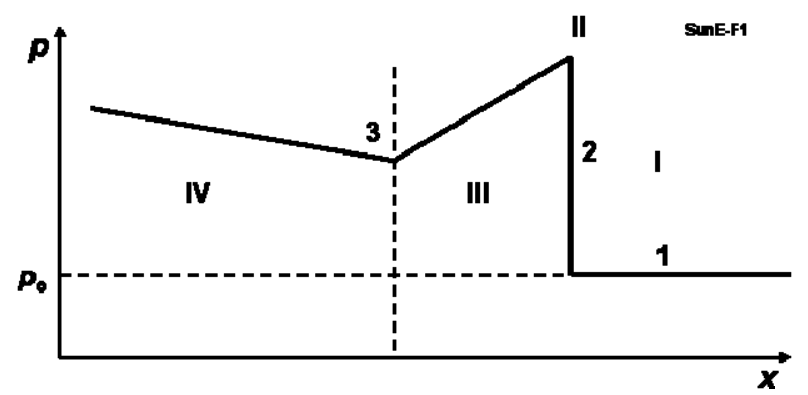

Figure 1. Pressure in detonation wave. I-plasma, II-front of detonation wave, III-zone of the initial thermonuclear fusion reaction, $\mathrm{IV}$ - products of reaction and next reaction, $p_{0}$-initial pressure, $x$-distance.

$$
D=\sqrt{2 Q\left(\gamma^{2}-1\right)}
$$

$\gamma \approx 1.2-1.4$ is adiabatic coefficient [13].

\section{Model of Artificial Sun Explosion. Estimation of Ignition}

Thermonuclear reactions proceeding in the Sun's core are under high temperature and pressure. However the core temperature is substantially lower than that needed to overcome the Columb barrier. That way the thermonuclear reaction is very slow and the Sun's life cycle is about 10 billion years. But that is enough output to keep the Sun a plasma ball, hot enough for life on Earth to exist. Now we are located in the middle of the Sun's life and have about 5 billions years until the Sun becomes a Red Giant.

However, this presumes that the Sun is stable against deliberate tampering. Supposing our postulations are correct, the danger exists that introducing a strong thermonuclear explosion into the Sun which is a container of fuel for thermonuclear reactions, the situation can be cardinally changed. For correct computations it is necessary to have a comprehensive set of full initial data (for example, all cross-section areas of all nuclear reactions) and supercomputer time. The author does not have access to such resources. That way he can only estimate probability of these reactions, their increasing or decreasing. Supportive investigations are welcome in order to restore confidence in humanity's long term future [14].

\section{AB-Criterion for Solar Detonation}

A self-supporting detonation wave is possible if the speed of detonation wave is greater or equals the ion sound speed:

$$
D \geq v, \text { where } D=\sqrt{2 Q\left(\gamma^{2}-1\right)}, v=\left(\frac{\gamma z k T_{k}}{m_{i}}\right)^{1 / 2} .
$$

Here $Q$ is a nuclear specific heat $[\mathrm{J} / \mathrm{kg}], \gamma=1.2-1.4$ is adiabatic coefficient (they are noted in (17)-(18)); $z$ is number of the charge of particle after fusion reaction $(z=$ 1 for $\left.{ }^{2} \mathrm{H}\right), k=1.36 \times 10^{-23}$ is Boltzmann constant, $\mathrm{J} / \mathrm{K} ; T_{k}$ is temperature of plasma after fusion reaction in Kelvin degrees; $m_{i}=\mu m_{p}$ is mass of ion after fusion reaction, $\mathrm{kg}$; $m_{p}=1.67 \times 10^{-27} \mathrm{~kg}$ is mass of proton; $\mu$ is relative mass, $\mu=2$ for ${ }^{2} \mathrm{H}$.

When we have sign " $>$ " the power of the detonation wave increases, when we have the sign " $<$ " it decreases.

Substitute two last equations in the first equation in (19) we get

$$
\begin{aligned}
& D^{2} \geq v^{2}, 2 Q\left(\gamma^{2}-1\right) \geq \frac{\gamma z k T_{k}}{m_{i}} . \\
& \text { where } Q=\frac{f e E \tau}{n m_{p}}=\frac{1}{4} n^{2} e E\langle\sigma v\rangle \frac{\tau}{n m_{p}}
\end{aligned}
$$

where $f$ is speed of nuclear reaction, $\mathrm{s} / \mathrm{m}^{3} ; e=1.6 \times 10^{-19}$ is coefficient for converting the energy from electronvolts to joules; $E$ is energy of reaction in $\mathrm{eV} ; n$ is number particles ( $p$ - protons) in $\mathrm{m}^{3} ;\langle\sigma v\rangle$ is reaction rate, $\mathrm{m}^{3} / \mathrm{s}$ (Figure 2), $m_{i}=2 m_{p}, \tau$ is time, sec.

From (20) we get the AB-Criterion for artificial Sun explosion:

$$
\begin{aligned}
n \tau & \geq \frac{\gamma z k T_{k}}{\left(\gamma^{2}-1\right) e E\langle\sigma v\rangle}=\frac{1.16 \times 10^{4} \gamma z k T_{e}}{\left(\gamma^{2}-1\right) e E\langle\sigma v\rangle} \\
& =\frac{\gamma z T_{e}}{\left(\gamma^{2}-1\right) E\langle\sigma v\rangle}
\end{aligned}
$$

where $T_{e}$ is temperature of plasma after reaction in $\mathrm{eV}$.

The offered AB-Criterion (21) is different from the well-known Lawson criterion

$$
n_{e} \tau_{e}>\frac{12 k_{B} T_{k}}{E_{c h}\langle\sigma v\rangle}
$$

where $E_{c h}$ is energy of reaction in $\mathrm{keV}, k_{B}$ is Boltzmann

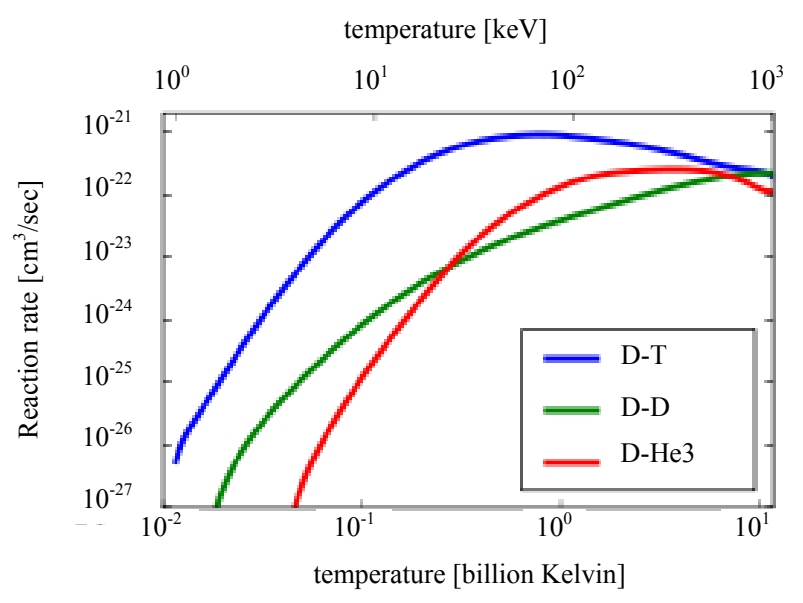

Figure 2. Reaction rate $\langle\sigma v\rangle$ via plasma temperature for D-T (top), D-D (middle) and D- ${ }^{3} \mathrm{He}$ (bottom in left side). 
constant.

The offered AB-Criterion contains the $\gamma$ adiabatic coefficient and $z$-number of electric charge in the electron charges. It is not surprising because Lawson derived his criterion from the condition where the energy of the reaction must be greater than the loss of energy by plasma into the reactor walls, where

$$
W_{\text {reaction }}>W_{\text {loss }} \text {. }
$$

In our case no the reactor walls and plasma reflects the any radiation.

The offered AB-Criterion is received from the condition (19): Speed of self-supporting detonation wave must be greater than the speed of sound where

$$
D>v \text {. }
$$

For main reaction $p+p$ the AB-Criterion (21) has a form

$$
n \tau \geq \frac{T_{e}}{E\langle\sigma v\rangle} .
$$

Estimation. Let us take the first step of the reaction ${ }^{1} \mathrm{H}$ $+{ }^{1} \mathrm{H}$ (12)-(13) having in point 3 (Figure 1) $T_{e}=10^{5} \mathrm{eV}$, $E \approx 1.44 \times 10^{6} \mathrm{eV},<\sigma \mathrm{v}>\approx \times 10^{-22}$. Substituting them in Equation (21) we receive

$$
n \tau>0.7 \times 10^{21} \text {. }
$$

The Sun surface (photosphere) has density $n=10^{23}$ $1 / \mathrm{m}^{3}$, the encounter time of protons in the hypothetical detonation wave III (Figure 1) may be over $0.01 \mathrm{sec}$. The values in left and right sides of (22) have the same order. That means a thermonuclear bomb exploded within the Sun may conceivably be able to serve as a detonator which produces a self-supported nuclear reaction and initiates the artificial explosion of the Sun.

After the initial reaction the temperature of plasma is very high $(>1 \mathrm{MeV})$ and time of next reaction may be very large (hundreds of seconds), the additional energy might in these conditions increase up to $26 \mathrm{MeV}$.

A more accurate computation is possible but will require cooperation of an interested supercomputer team with the author, or independent investigations with similar interests [15].

\section{Penetration of Thermonuclear Bomb into Sun}

The Sun is a ball of plasma (ionized gases), not a solid body. A properly shielded thermonuclear bomb can permeate deep into the Sun. The warhead may be protected on its' way down by a special high reflectivity mirror offered, among others, by author A.A. Bolonkin in 1983 [11] and described in [7] Chapters 12, 3A, [8] Ch.5 (see also [9-15]). This mirror allows to maintain a low temperature of the warhead up to the very boundary of the solar photosphere. At that point its' velocity is gigantic, about $617.6 \mathrm{~km} / \mathrm{s}$, assuring a rapid penetration for as far as it goes.

The top solar atmosphere is very rarefied; a milliard (US billion) times less than the Earth's atmosphere. The Sun's photosphere has a density approximately 200 times less than the Earth's atmosphere. Some references give a value of only $0.0000002 \mathrm{gm} / \mathrm{cm}^{3}(0.1$ millibar $)$ at the photosphere surface. Since present day ICBM warheads can penetrate down (by definition) to the 1 bar level (Earth's surface) and that is by no means the boundary of the feasible, the 10 bar level may be speculated to be near-term achievable. The most difficult entry yet was that of the Galileo atmospheric probe on Dec. 7, 1995 [16]. The Galileo Probe was a $45^{\circ}$ sphere-cone that entered Jupiter's atmosphere at $47.4 \mathrm{~km} / \mathrm{s}$ (atmosphere relative speed at $450 \mathrm{~km}$ above the 1 bar reference altitude). The peak deceleration experienced was $230 \mathrm{~g}\left(2.3 \mathrm{~km} / \mathrm{s}^{2}\right)$. Peak stagnation point pressure before aeroshell jettison was 9 bars $(900 \mathrm{kPa})$. The peak shock layer temperature was approximately $16000 \mathrm{~K}$ (and remember this is into hydrogen (mostly) the solar photosphere is merely 5800 K). Approximately $26 \%$ of the Galileo Probe's original entry mass of $338.93 \mathrm{~kg}$ was vaporized during the 70 second heat pulse. Total blocked heat flux peaked at approximately $15000 \mathrm{~W} / \mathrm{cm}^{2}$ (hotter than the surface of the Sun).

If the entry vehicle was not optimized for slowdown as the Galileo Probe but for penetration like a modern ICBM warhead, with extra ablatives and a sharper cone half-angle, achievable penetration would be deeper and faster. If 70 seconds atmospheric penetration time could be achieved, (with minimal slowdown) perhaps up to $6 \%$ of the way to the center might be achieved by near term technology.

The outer penetration shield of the warhead may be made from carbon (which is an excellent ablative heat protector). The carbon is also an excellent nuclear catalyst of the nuclear reactions in the $\mathrm{CNO}$ solar thermonuclear cycle and may significantly increase the power of the initial explosion [17].

A century hence, what level of penetration of the solar interior is possible? This depth is unknown to the author, exceeding plausible engineering in the near term. Let us consider a hypothetical point (top of the radiation layer) 30 percent of the way from the surface to the core, at the density of $0.2 \mathrm{~g} / \mathrm{cm}^{3}$ with a temperature of $2,000,000^{\circ} \mathrm{C}$. No material substance can withstand such heat-for extended periods.

We may imagine however hypothetical penetration aids, analogous to ICBM techniques of a half century ago. Shock waves bear the brunt of the encountered heat and force it aside, the opacity shielding the penetrator. A form of multiple disposable shock cones may be em- 
ployed to give the last in line a chance to survive; indeed the destruction of the next to last may arm the trigger.

If the heat isolation shield and multiple penetration aids can protect the bomb at near entry velocity for a hellish 10 minute interval, (which to many may seem impossible but which cannot be excluded without definitive study-remember we are speaking now of centuries hence, not the near term case above-see reference 14) that means the bomb may reach the depth of 350 thousands kilometers or $0.5 R$, where $\mathrm{R}=696 \times 103 \mathrm{~km}$ is Sun's radius.

The Sun density via relative Sun depth may be estimated by the equation

$$
n=n_{s} e^{20.4 \bar{h}}, \text { where } \bar{h}=h / R,
$$

where $n_{s} \approx 10^{23} 1 / \mathrm{m}^{3}$ is the plasma density on the photosphere surface; $\mathrm{h}$ is deep, $\mathrm{km}$; $\mathrm{R}=696 \times 10^{3}$ is solar radius, $\mathrm{km}$. At a solar interior depth of $h=0.5 R$ the relative density is greater by 27 thousand times than on the Sun's surface.

Here the density and temperature are significantly more than on the photosphere's surface. And conditions for the detonation wave and thermonuclear reaction are "better"- from the point of view of the attacker.

\section{Estimation of Nuclear Bomb Needed for Sun Explosion}

Sound speed into plasma headed up $\mathrm{T}=100{ }^{\circ} \mathrm{K}$ million degrees is about

$$
v \approx 10^{2} T^{0.5} \mathrm{~m} / \mathrm{s}=10^{6} \mathrm{~m} / \mathrm{s} .
$$

Time of nuclear explosion (a full nuclear reaction of bomb) is less $t=10^{-4} \mathrm{sec}$. Therefore the radius of heated Sun photosphere is about $R=v t=100 \mathrm{~m}$, volume $V$ is about

$$
V=\frac{4}{3} \pi R^{3} \approx 4 \times 10^{6} \mathrm{~m}^{3} .
$$

Density of Sun photosphere is $p=2 \times 10^{-4} \mathrm{~kg} / \mathrm{m}^{3}$. Consequently the mass of the heated photosphere is about $m=p V=1000 \mathrm{~kg}$.

The requested power of the nuclear bomb for heating this mass for temperature $T=10^{4} \mathrm{eV}(100 \mathrm{~K}$ million degrees) is approximately

$$
\begin{aligned}
E & =10^{3} \times 10^{4} / 1.67 \times 10^{-27} \mathrm{eV} \approx 0.6 \times 10^{34} \mathrm{eV} \\
& \approx 2 \times 10^{15} \mathrm{~J} \approx 0.5 \mathrm{Mt}
\end{aligned}
$$

The requested power of nuclear bomb is about 0.5 Megatons. The average power of the current thermonuclear bomb is $5-10 \mathrm{Mt}$. That means the current thermonuclear bomb may be used as a fuse of Sun explosion. That estimation needs in a more complex computation by a power computer.

\section{Results of Research}

The Sun contains $73.46 \%$ hydrogen by weight. The isotope hydrogen-1 $(99.985 \%$ of hydrogen in nature) is usable fuel for a fusion thermonuclear reaction.

The $p$-p reaction runs slowly within the Sun because its temperature is low (relative to the temperatures of nuclear reactions). If we create higher temperature and density in a limited region of the solar interior, we may be able to produce self-supporting, more rapid detonation thermonuclear reactions that may spread to the full solar volume. This is analogous to the triggering mechanisms in a thermonuclear bomb. Conditions within the bomb can be optimized in a small area to initiate ignition, build a spreading reaction and then feed it into a larger area, allowing producing a "solar hydrogen bomb" of any power-but not necessarily one whose power can be limited. In the case of the Sun certain targeting practices may greatly increase the chances of an artificial explosion of the entire Sun. This explosion would annihilate the Earth and the Solar System, as we know them today.

Author A.A. Bolonkin has researched this problem and shown that an artificial explosion of Sun cannot be precluded. In the Sun's case this lacks only an initial fuse, which induces the self-supporting detonation wave. This research has shown that a thermonuclear bomb exploded within the solar photosphere surface may be the fuse for an accelerated series of hydrogen fusion reactions.

The temperature and pressure in this solar plasma may achieve a temperature that rises to billions of degrees in which all thermonuclear reactions are accelerated by many thousands of times. This power output would further heat the solar plasma. Further increasing of the plasma temperature would, in the worst case, climax in a solar explosion.

The possibility of initial ignition of the Sun significantly increases if the thermonuclear bomb is exploded under the solar photosphere surface. The incoming bomb has a diving speed near the Sun of about $617 \mathrm{~km} / \mathrm{sec}$. Warhead protection to various depths may be feasibleablative cooling which evaporates and protects the warhead some minutes from the solar temperatures. The deeper the penetration before detonation the temperature and density achieved greatly increase the probability of beginning thermonuclear reactions which can achieve explosive breakout from the current stable solar condition.

Compared to actually penetrating the solar interior, the flight of the bomb to the Sun, (with current technology requiring a gravity assist flyby of Jupiter to cancel the solar orbit velocity) will be easy to shield from both radiation and heating and melting. Numerous authors, including A. A. Bolonkin in works [7-12] offered and showed the high reflectivity mirrors which can protect 
the flight article within the orbit of Mercury down to the solar surface.

The author A. A. Bolonkin originated the $\mathrm{AB}$ Criterion, which allows estimating the condition required for the artificial explosion of the Sun.

\section{Discussion}

If we (humanity - unfortunately in this context, an insane dictator representing humanity for us) create a zone of limited size with a high temperature capable of overcoming the Coulomb barrier (for example by insertion of a specialized thermonuclear warhead) into the solar photosphere (or lower), can this zone ignite the Sun's photosphere (ignite the Sun's full load of thermonuclear fuel)? Can this zone self-support progressive runaway reaction propagation for a significant proportion of the available thermonuclear fuel?

If it is possible, researchers can investigate the problems: What will be the new solar temperature? Will this be metastable, decay or runaway? How long will the transformed Sun live, if only a minor change? What the conditions will be on the Earth during the interval, if only temporary? If not an explosion but an enhanced burn results the Sun might radically increase in luminosity for-say-a few hundred years. This would suffice for an average Earth temperature of hundreds of degrees over $0^{\circ} \mathrm{C}$. The oceans would evaporate and Earth would bake in a Venus like greenhouse, or even lose its' atmosphere entirely.

It would not take a full scale solar explosion, to annihilate the Earth as a planet for Man. (For a classic report on what makes a planet habitable, co-authored by Issac Asimov, see http://www.rand.org/pubs/commercial_books/ 2007/RAND_CB179-1.pdf).

Converting the sun even temporarily into a "superflare" star, (which may hugely vary its output by many percent, even many times) over very short intervals, not merely in heat but in powerful bursts of shorter wavelengths) could kill by many ways, notably ozone depletion - thermal stress and atmospheric changes and hundreds of others of possible scenarios - in many of them, human civilization would be annihilated. And in many more, humanity as a species would come to an end.

The reader naturally asks: Why even contemplate such a horrible scenario? It is necessary because as thermonuclear and space technology spreads to even the least powerful nations in the centuries ahead, a dying dictator having thermonuclear missile weapons can produce (with some considerable mobilization of his military/industrial complex) - the artificial explosion of the Sun and take into his grave the whole of humanity. It might take tens of thousands of people to make and launch the hardware, but only a very few need know the final targeting data of what might be otherwise a weapon purely thought of (within the dictator's defense industry) as being built for peaceful, deterrent use.

Those concerned about Man's future must know about this possibility and create some protective system-or ascertain on theoretical grounds that it is entirely impossible, which would be comforting.

Suppose, however that some variation of the following is possible, as determined by other researchers with access to good supercomputer simulation teams. What, then is to be done?

The action proposed depends on what is shown to be possible.

Suppose that no such reaction is possible-it dampens out unnoticeably in the solar background, just as no fission bomb triggered fusion of the deuterium in the oceans proved to be possible in the Bikini test of 1946 . This would be the happiest outcome.

Suppose that an irruption of the Sun's upper layers enough to cause something operationally similar to a targeted "coronal mass ejection"-CME- of huge size targeted at Earth or another planet? Such a CME like weapon could have the effect of a huge electromagnetic pulse. Those interested should look up data on the 1859 solar superstorm, the Carrington event, and the Stewart Super Flare. Such a CME/EMP weapon might target one hemisphere while leaving the other intact as the world turns. Such a disaster could be surpassed by another step up the escalation ladder - by a huge hemisphere killing thermal event of $\sim 12$ hours duration such as postulated by science fiction writer Larry Niven in his 1971 story "Inconstant Moon"-apparently based on the Thomas Gold theory (ca. 1969-70) of rare solar superflares of 100 times normal luminosity. Subsequent research ${ }^{18}$ (Wdowczyk and Wolfendale, 1977) postulated horrific levels of solar activity, ozone depletion and other such consequences might cause mass extinctions. Such an improbable event might not occur naturally, but could it be triggered by an interested party? A triplet of satellites monitoring at all times both the sun from Earth orbit and the "far side" of the Sun from Earth would be a good investment both scientifically and for purposes of making sure no "creative" souls were conducting trial CME eruption tests!

Might there be peaceful uses for such a capability? In the extremely hypothetical case that a yet greater super-scale CME could be triggered towards a given target in space, such a pulse of denser than naturally possible gas might be captured by a giant braking array designed for such a purpose to provide huge stocks of hydrogen and helium at an asteroid or moon lacking these materials for purposes of future colonization.

A worse weapon on the scale we postulate might be an asymmetric eruption (a form of directed thermonuclear 
blast using solar hydrogen as thermonuclear fuel), which shoots out a coherent (in the sense of remaining together) burst of plasma at a given target without going runaway and consuming the outer layers of the Sun. If this quite unlikely capability were possible at all (dispersion issues argue against it - but before CMEs were discovered, they too would have seemed unlikely), such an apocalyptic "demo" would certainly be sufficient emphasis on a threat, or a means of warfare against a colonized solar system. With a sufficient thermonuclear burn - and if the condition of nondispersion is fulfilled - might it be possible to literally strip a planet—Venus, say—of its' atmosphere? (It might require a mass of fusion fuel - and a hugely greater non-fused expelled mass comparable in total to the mass to be stripped away on the target planet.)

It is not beyond the limit of extreme speculation to imagine an expulsion of this order sufficient to strip Jupiter's gas layers off the "Super-Earth" within.-To strip away $90 \%$ or more of Jupiter's mass (which otherwise would take perhaps $\sim 400$ Earth years of total solar output to disassemble with perfect efficiency and neglecting waste heat issues). It would probably waste a couple Jupiter masses of material (dispersed hydrogen and helium). It would be an amazing engineering capability for long term space colonization, enabling substantial uses of materials otherwise unobtainable in nearly all scenarios of long term space civilization.

Moving up on the energy scale - "boosting" or "damping" a star, pushing it into a new metastable state of greater or lesser energy output for times not short compared with the history of civilization, might be a very welcome capability to colonize another star system - and a terrifying reason to have to make the trip.

And of course, in the uncontrollable case of an induced star explosion, in a barren star system it could provide a nebula for massive mining of materials to some future super-civilization. It is worth noting in this connection that the Sun constitutes 99.86 percent of the material in the Solar System, and Jupiter another.1 percent. Literally a thousand Earth masses of solid (iron, carbon) building materials might be possible, as well as thousands of oceans of water to put inside space colonies in some as yet barren star system.

But here in the short-term future, in our home solar system, such a capability would present a terrible threat to the survival of humanity, which could make our own solar system completely barren.

The list of possible countermeasures does not inspire confidence. A way to interfere with the reaction (dampen it once it starts)? It depends on the spread time, but seems most improbable. We cannot even stop nuclear reactions once they take hold on Earth - the time scales are too short.

Is defense of the Sun possible? Unlikely—such a task makes missile defense of the Earth look easy. Once a gravity assist Jupiter flyby nearly stills the velocity with which a flight article orbits the Sun, it will hang relatively motionless in space and then begin the long fall to fiery doom. A rough estimate yields only one or two weeks to intercept it within the orbit of Mercury, and the farther it falls the faster it goes, to science fiction-like velocities sufficient to reach Pluto in under six weeks before it hits.

A perimeter defense around the Sun? The idea seems impractical with near term technology.

The Sun is a hundred times bigger sphere than Earth in every dimension. If we have 10,000 ready to go interceptor satellites with extreme sunshields that function a few solar radii out each one must be able to intercept with $99 \%$ probability the brightening light heading toward its' sector of the Sun over a circle the size of Earth, an incoming warhead at around $600 \mathrm{~km} / \mathrm{sec}$.

If practical radar range from a small set is considered (4th power decline of echo and return) as 40,000 km then only 66 seconds would be available to plot a firing solution and arm for a destruct attempt. More time would be available by a telescope looking up for brightening, infalling objects - but there are many natural incoming objects such as meteors, comets, etc. A radar might be needed just to confirm the artificial nature of the in-falling object (given the short actuation time and the limitations of rapid storable rocket delta-v some form of directed nuclear charge might be the only feasible countermeasure) and any leader would be reluctant to authorize dozens of nuclear explosions per year automatically (there would be no time to consult with Earth, eight lightminutes away — and eight more back, plus decision time). But the cost of such a system, the reliability required to function endlessly in an area in which there can presumably be no human visits and the price of its' failure, staggers the mind. And such a "thin" system would be not difficult to defeat by a competent aggressor...

A satellite system near Earth for destroying the rockets moving to the Sun may be a better solution, but with more complications, especially since it would by definition also constitute an effective missile defense and space blockade. Its' very presence may help spark a war. Or if only partially complete but under construction, it may invite preemption, perhaps on the insane scale that we here discuss...

Astronomers see the explosion of stars. They name these stars novae and supernovae- - New Stars" and try to explain (correctly, we are sure, in nearly all cases) their explosion by natural causes. But some few of them, from unlikely spectral classifications, may be result of war between civilizations or fanatic dictators inflicting their final indignity upon those living on planets of the given star. We have enough disturbed people, some in 
positions of influence in their respective nations and organizations and suicide oriented violent people on Earth. But a nuclear bomb can destroy only one city. A dictator having possibility to destroy the Solar System as well as Earth can blackmail all countries - even those of a future Kardashev scale 2 star-system wide civilization-and dictate his will/demands on any civilized country and government. It would be the reign of the crazy over the sane.

Author A.A. Bolonkin already warned about this possibility in 2007 (see his interview http://www.pravda.ru/ science/planet/space/05-01-2007/208894-sun_detonation -0 [15] (in Russian) (A translation of this is appended at the end of this article) and called upon scientists and governments to research and develop defenses against this possibility. But some people think the artificial explosion of Sun impossible. This led to this current research to give the conditions where such detonations are indeed possible. That shows that is conceivably possible even at the present time using current rockets and nuclear bombs - and only more so as the centuries pass. Let us take heed, and know the risks we face-or disprove them.

The first information about this work was published in [15]. This work produced the active Internet discussion in [18]. Among the raised questions were the following:

1) It is very difficult to deliver a warhead to the Sun. The Earth moves relative to the Sun with a orbital velocity of $30 \mathrm{~km} / \mathrm{s}$, and this speed should be cancelled to fall to the Sun. Current rockets do not suffice, and it is necessary to use gravitational maneuvers around planets. For this reason (high delta- $\mathrm{V}$ (velocity changes required) for close solar encounters, the planet Mercury is so badly investigated (probes there are expensive to send).

Answer: The Earth has a speed of $29 \mathrm{~km} / \mathrm{s}$ around the Sun and an escape velocity of only $11 \mathrm{~km} / \mathrm{s}$. But Jupiter has an orbital velocity of only $13 \mathrm{~km} / \mathrm{sec}$ and an escape velocity of $59.2 \mathrm{~km} / \mathrm{s}$. Thus, the gravity assist Jupiter can provide is more than the Earth can provide, and the required delta- $v$ at that distance from the Sun far lessenough to entirely cancel the sun-orbiting velocity around the Sun, and let it begin the long plunge to the Solar orb at terminal velocity achieving Sun escape speed 617.6 $\mathrm{km} / \mathrm{s}$. Notice that for many space exploration maneuvers, we require a flyby of Jupiter, exactly to achieve such a gravity assist, so simply guarding against direct launches to the Sun from Earth would be futile!

2) Solar radiation will destroy any a probe on approach to the Sun or in the upper layers of its photosphere.

Answer: It is easily shown, the high efficiency AB-reflector can full protection the apparatus. See [7] Chapters 12, 3A, [8] Ch.5, (see also [9-12].

3) The hydrogen density in the upper layers of the photosphere of the Sun is insignificant, and it would be much easier to ignite hydrogen at Earth oceans if it in general is possible.

Answer: The hydrogen density is enough known. The Sun has gigantic advantage - that is PLASMA. Plasma of sufficient density reflects or blocks radiation-it has opacity. That means: no radiation losses in detonation. It is very important for heating. The AB Criterion in this paper is received for PLASMA. Other planets of Solar system have MOLECULAR atmospheres which passes radiation. No sufficient heating - no detonation! The water has higher density, but water passes the high radiation (for example $\gamma$-radiation) and contains a lot of oxygen (89\%), which may be bad for the thermonuclear reaction. This problem needs more research.

\section{Summary}

This is only an initial investigation. Detailed supercomputer modeling which allows more accuracy would greatly aid prediction of the end results of a thermonuclear explosion on the solar photosphere.

Author invites the attention of scientific society to detailed research of this problem and devising of protection systems if it proves a feasible danger that must be taken seriously. The other related ideas author Bolonkin offers in [5-15].

\section{Acknowledgements}

The author wishes to acknowledge Alexei Turchin (Russia) for discussing the problems in this article [18].

\section{REFERENCES}

[1] AIP Physics Desk Reference, 3rd Edition, Spring, Berlin, 888 Pages.

[2] I. Grigoriev, "Handbook of Physical Quantities," CRC Press, Boca Raton, 1997.

[3] I. K. Kikoin, "Tables of Physical Values," Atomizdat, Moscow Ctiy, 1975, 1006 Pages (in Russian).

[4] K. Nishikawa and M. Wakatani, "Plasma Physics," Spring, Berlin, 2000. doi:10.1007/978-3-662-04078-2

[5] A. A. Bolonkin, "New AB-Thermonuclear Reactor for Aerospace," AIAA-2006-7225 to Space-2006 Conference, San Jose, 19-21 September 2006. http://arxiv.org/ftp/arxiv/papers/0706/0706.2182.pdf http://arxiv.org/ftp/arxiv/papers/0803/0803.3776.pdf

[6] A. A. Bolonkin, "Simplest AB-Thermonuclear Space Propulsion and Electric Generator". http://arxiv.org/ftp/physics/papers/0701/0701226.pdf

[7] A. A. Bolonkin, "Non-Rocket Space Launch and Flight," Elsevier, Amsterdam, 2006, 488 Pages (The Book Contains Theories of the More than 20 New Revolutionary Author Ideas in Space and Technology). http://Bolonkin.narod.ru/p65.htm http://www.scribd.com/doc/24056182 
[8] A. A. Bolonkin, "New Concepts, Ideas and Innovations in Aerospace and Technology, Nova, 2007," The Book Contains Theories of the More than 20 New Revolutionary Author Ideas in Space and Technology. http://Bolonkin.narod.ru/p65.htm http://www.scribd.com/doc/24057071

[9] A. A. Bolonkin and R. B. Cathcart, "Macro-Projects: Environment and Technology," NOVA, 2009, 536 Pages. http://Bolonkin.narod.ru/p65.htm

Book contains many new revolutionary ideas and projects. http://www.scribd.com/doc/24057930.

[10] A. A. Bolonkin, "High Speed AB Solar Sail," 42 JOINT Propulsion Conferences, Sacramento, 9-12 July 2006. http://arxiv.org/ftp/physics/papers/0701/0701073.pdf

[11] A. A. Bolonkin, "Light Multi-Reflex Engine," Journal of British Interplanetary Society, Vol. 57, No. 9-10, 2004, pp. 353-359.

[12] A. A. Bolonkin, "Light Pressure Engine, Patent (Author Certificate) \# 1183421, 1985, USSR (Priority on 5 January 1983).

[13] A. A. Bolonkin, "Converting of Matter to Nuclear Energy by AB-Generator," American Journal of Engineering and Applied Science, Vol. 2, No. 2, 2009, pp. 683-693. http://www.scipub.org/fulltext/ajeas/ajeas24683-693.pdf http://www.scribd.com/doc/24048466/

[14] A. A. Bolonkin, "Femtotechnology. Nuclear AB-Matter with Fantastic Properties," American Journal of Engineering and Applied Sciences, Vol. 2, No. 2, 2009, pp. 501514.

http://www.scipub.org/fulltext/ajeas/ajeas22501-514.pdf http://www.scribd.com/doc/24046679/

[15] A. A. Bolonkin, "Artificial Explosion of Sun," 2007 (in Russian)

http://www.pravda.ru/science/planet/space/05-01-2007/20 8894-sun_detonation-0

[16] Solar Physics Group at NASA's Marshall Space Flight Center Website for Solar Facts. http://solarscience.msfc.nasa.gov/

[17] J. Wdowczyk and A. W. Wolfendale, "Cosmic Rays and Ancient Catastrophes," Nature, Vol. 268, 1977, Article ID: 510 . http://www.nature.com/nature/journal/v268/n5620/abs/26 8510a0.html

[18] A. V. Turchin, "The Possibility of Artificial Fusion Explosion of Giant Planets and Other Objects of Solar System," 2009. http://www.scribd.com/doc/8299748/Giant-planets-igniti on 